\title{
Autologous fat grafting in breast reconstruction: implications for follow-up and surveillance
}

\author{
Summer E. Hanson ${ }^{1}$, Sahil K. Kapur ${ }^{1}$, Rosa F. Hwang ${ }^{2}$, Mark S. Dryden ${ }^{3}$ \\ ${ }^{1}$ Department of Plastic Surgery, ${ }^{2}$ Department of Breast Surgical Oncology, ${ }^{3}$ Department of Diagnostic Radiology - Breast Imaging Section, The \\ University of Texas MD Anderson Cancer Center, Houston, TX, USA \\ Contributions: (I) Concept and design: All authors; (II) Administrative support: All authors; (III) Provision of study materials or patients: All authors; \\ (IV) Collection and assembly of data: All authors; (V) Data analysis and interpretation: All authors; (VI) Manuscript writing: All authors; (VII) Final \\ approval of manuscript: All authors. \\ Correspondence to: Summer E. Hanson, MD, PhD, FACS. Associate Professor, Director of Translational Research, Department of Plastic Surgery, \\ The University of Texas MD Anderson Cancer Center, 1400 Pressler Street, Unit 1488, Houston, TX 77030, USA. \\ Email: sehanson@mdanderson.org.
}

\begin{abstract}
The procedural volume of autologous fat grafting (AFG) has risen over the past several years, specifically in the setting of breast reconstruction, despite controversy surrounding its oncologic safety. While some in vitro and animal models have cast doubt on the oncologic safety of AFG, there is no clinical evidence that AFG in breast reconstruction is associated with an increased risk of cancer development or recurrence or an inability to adequately assess changes in the breast. That being said, recommendations regarding surveillance and follow-up after AFG in breast reconstruction are largely surgeon directed and differ across practices. The purpose of this review is to summarize the current literature and provide evidence-based recommendations.
\end{abstract}

Keywords: Autologous fat grafting (AFG); breast reconstruction; lipotransfer; oncologic safety

Submitted Mar 02, 2020. Accepted for publication Mar 18, 2020.

doi: 10.21037 /gs.2020.04.04

View this article at: http://dx.doi.org/10.21037/gs.2020.04.04

\section{Introduction}

The procedural volume of autologous fat grafting (AFG) in breast surgery, both cosmetic and reconstructive, continues to increase, according to an American Society of Plastic Surgeons (ASPS) procedural statistics report (1). In AFG for breast reconstruction, adipose tissue is harvested from one site of the body, usually from the abdomen or flanks, via lipoaspiration, processed or purified to remove blood, free lipids, and other cellular debris, and delivered to the breast to address contour irregularities, volume deficits, and asymmetry. Although AFG is not a new concept, the indications and techniques have evolved over the past few decades (2-4). Despite a better understanding of the optimal processing and delivery of adipose grafts, there remains low predictability of graft retention and questionable safety in the setting of oncologic breast reconstruction. Therefore, recommendations regarding surveillance and follow-up after AFG in breast reconstruction are largely surgeon directed and differ across practices. The purpose of this review is to summarize the current literature and provide recommendations based on our practice.

\section{AFG}

In AFG, engraftment of adipose tissue relies on the local tissue environment for new vessel growth. There are a few proposed mechanisms of engraftment-mainly, cell replacement and cell supplementation-with identified distinct areas of adipocyte survival, a regenerating zone of ischemic adipocytes replaced by ASCs, and a central area of necrosis replaced by scarring $(5,6)$. Even under the best conditions, the architecture of the breast is altered. As with any tissue transfer, there are several steps in the process that 
Table 1 Summary of radiographic findings following fat grafting in cosmetic breast surgery

\begin{tabular}{lccc}
\hline Radiographic finding & Mammogram \% (95\% Cl) & Ultrasound \% (95\% Cl) & MRI \% (95\% Cl) \\
\hline Fat necrosis & $14 \%(11.4-16.6)$ & $5.7 \%(2.9-8.5)$ & $7.7 \%(4-11.4)$ \\
Oil cyst & $12.3 \%(10.5-14.1)$ & $5.1 \%(2-8.2)$ & $1.7 \%(0-3.4)$ \\
Macrocalcifications & $7 \%(3.8-10.2)$ & $5.3 \%(2.7-7.9)$ & $1.5 \%(0.3-4.5)$ \\
Microcalcifications & $9 \%(6.4-11.5)$ & $4.8 \%(2.8-6.7)$ & $1.9 \%(0.1-7.3)$ \\
"Scar" & $12.9 \%(1.1-24.7)$ & - & -
\end{tabular}

Summary of pooled analysis (29). Cl, Confidence interval; MRI, magnetic resonance imaging.

may affect the viability of the tissue.

Adipose tissue grafts add soft tissue volume through not only mature adipocytes but also potentially abundant progenitor cells such as adipose-derived stem cells (ASCs) and associated cytokines within the tissue stroma $(7,8)$. A 2009 task force statement from the ASPS highlighted concerns about AFG in breast reconstruction, including fears that ASCs and cytokines would stimulate cancer growth and increase cancer recurrence or that the grafts would lead to fat necrosis or calcifications that may interfere with mammography and subsequent cancer detection (9). While there is no level I evidence assessing the safety of AFG in breast reconstruction, several retrospective studies have demonstrated no increased risk of new breast cancer, locoregional recurrence (LRR), or metastatic disease with AFG (10-17). Furthermore, there is evidence supporting beneficial effects of AFG in reconstructing irradiated tissue, by improving fibrosis, and reducing chest wall pain after breast cancer treatment, although the mechanisms behind these clinical findings have not been fully elucidated and are likely multi-factorial (18-21).

\section{Impact of AFG on imaging}

Breast surgery inherently alters the tissue parenchyma and may lead to aberrant imaging. Particularly in the setting of AFG to the breast, ischemia and resulting inflammation can cause fat necrosis, palpable abnormalities, calcifications, or oil cysts-all of which will potentially appear on radiographic imaging. Although radiographic changes have not been included in all of the AFG outcomes studies, there is sufficient data available to identify the most commonly seen changes in breast imaging after AFG (22-28). Table 1 summarizes the radiographic findings adapted from a systematic review by Groen et al. (29). Analysis of pooled data from 22 studies including 3,565 patients with follow-up ranging from 12 to 136 months demonstrates the most common mammographic findings after AFG to a normal breast are fat necrosis (14\%) and "scar" (12.9\%). Calcifications, both macro and micro, were observed in fewer than $10 \%$ of patient images [7\%, 95\% confidence interval (CI): 3.8-10.2; 9\%, 95\% CI: 6.4-11.5, respectively] and were generally distinguishable from those indicative of malignancy. The mean graft retention rate was $62.4 \%(29)$.

In a small retrospective review of 20 patients who underwent AFG in aesthetic breast surgery (23), the authors found no significant difference in breast tissue density before and after lipomodeling on pre-operative and post-operative mammograms. Similarly, AFG did not change the Breast Imaging Reporting and Data System (BIRADS) score. Postgraft mammography demonstrated a calcification rate of $16 \%$ and an oil cyst rate of $25 \%$. Rubin and colleagues (24) performed a comparison study in which academic breast imaging specialists were blinded to mammograms of patients who had undergone either cosmetic augmentation with AFG or breast reduction mammoplasty (BR). The authors found statistically significant differences in rates of "scarring" $(17.6 \%$ versus $85.5 \%, \mathrm{P}<0.001)$ and presence of a mass or distortion warranting biopsy (2.8\% versus $13.6 \%, \mathrm{P}<0.001)$, with lower rates in the AFG group. Furthermore, BIRADS scores were more commonly negative (BIRADS 1 ) or benign (BIRADS 2) in the AFG group (88.9\% versus $75.6 \%$ ), and biopsy was more commonly recommended in the BR group (15.2\% versus $6.5 \%)$. Average follow-up recommendations were annually for the AFG group and within 6 months for the BR cohort (24). Taken together, these studies demonstrate that in the setting of cosmetic surgery for healthy breasts, AFG resulted in benign structural changes of the breast that had a lower incidence and were less severe compared with changes owing to breast reshaping surgery such as reduction mammoplasty. 
Table 2 Summary of radiographic findings following fat grafting in oncologic breast reconstruction

\begin{tabular}{lccc}
\hline Radiographic finding & Mammogram \% (95\% Cl) & Ultrasound \% (95\% Cl) & MRI \% (95\% Cl) \\
\hline Fat necrosis & $9 \%(4.2-13.9)$ & $11.3 \%(6.6-16)$ & $7.3 \%(4-10.3)$ \\
Oil cyst & $14.3 \%(12.2-16.4)$ & $26.7 \%(20.8-32.6)$ & $21.4 \%(17.1-25.7)$ \\
Macrocalcifications & $8.7 \%(3.3-14.5)$ & $1.3 \%(0.1-4.9)$ & - \\
Microcalcifications & $5.4 \%(2.3-8.5)$ & $1.3 \%(0.3-4.1)$ & - \\
Nodules or "Irregular lumps" & $5.1 \%(1.3-15.1)$ & - & - \\
Biopsy & $3.7 \%(0.1-7.2)$ & $3.7 \%(0.4-13.9)$ & - \\
Recurrence & $4.2 \%(0-23.8)$ & - & $-19.5)$ \\
\hline
\end{tabular}

Summary of pooled analysis (30). Cl, Confidence interval; MRI, magnetic resonance imaging.

In the oncologic setting, one would anticipate similar structural changes after AFG. Table 2 summarizes radiographic findings following $\mathrm{AFG}$ to the breast in oncologic reconstruction (30). Pooled analysis from 43 studies including 6,260 patients with follow-up ranging from 12 to 136 months demonstrates low overall complication rates for AFG, with high patient and surgeon satisfaction. LRR and distant recurrence (DR) rates after AFG in the oncologic setting were comparable to those in the general population with no lipofilling. Oil cysts were the most common radiographic finding on all imaging modalities in this systematic review, and the mean biopsy rate was only $3.7 \%$, with reported rates ranging from $1 \%$ to $24 \%$. General practice for follow-up imaging after breast reconstruction or breast conservation therapy (BCT) varies from case to case and institution to institution (31). Grafted adipose tissue appears lucent on follow-up mammograms but may lead to the development of calcifications consistent with fat necrosis, prompting biopsy or follow-up visits at shorter intervals than routine surveillance (32).

\section{Oncologic considerations}

Many studies have reported on the efficacy of fat grafting to the breast; however, only recently have there been studies with the patient numbers and length of follow-up to begin to address the oncologic safety of AFG in breast reconstruction, including several recent meta-analyses $(30,33,34)$. While there was considerable heterogeneity among these referenced studies, they demonstrate no clear statistical correlation between cancer recurrence and AFG. Agha and colleagues found a recurrence rate of $4.4 \%$ over the median follow-up period of 22.6 months (risk ratio $=1.33 ; \mathrm{P}=0.62 ; 95 \%$ CI: -0.43-4.09) (33), while Groen et al. reported that the LRR after AFG was 2.5\% (95\% CI: $1.7-3.7)$ and the DR rate was $2 \%$ (95\% CI: $1.1-3.5)$ (30). Further analysis of the comparative studies included demonstrates an overall complication rate of $8.4 \%$ (95\% CI: 7.6-9.1), with palpable nodules being the most common (11.5\%; 95\% CI: 9.0-13.9). Additional qualitative analyses of aesthetic, functional, patient-reported, and technical outcomes were, in general, non-standardized, self-reported, and overall positive.

The most recent analysis pooled 5,550 patients from 11 eligible studies and found no increased risk of LRR with AFG compared to the control cohort [odds ratio (OR): 0.71; 95\% CI: 0.47-1.04; $\mathrm{P}=0.08$ ] (34). This conclusion held true for subgroup analyses as well, including no increased risk with $\mathrm{AFG}$ specifically in mastectomy (OR: 0.74; 95\% CI: $0.41-1.33 ; \mathrm{P}=0.31$ ) or BCT (OR: 0.71; 95\% CI: $0.37-1.34 ; \mathrm{P}<0.29)$, and no increased risk with AFG specifically in invasive cancer (OR: 0.74; $95 \%$ CI: 0.41-1.33; $\mathrm{P}=0.31$ ) versus in situ disease (OR: 1.16 ; $95 \%$ CI: 0.18-7.38; $\mathrm{P}=0.55)$. A multi-centered, case-cohort study of patients who had mastectomy with immediate breast reconstruction found no increased risk of cancer recurrence with AFG compared to controls after adjusting for age, tumor stage, body mass index, and receptor status (hazard ratio: 0.97; 95\% CI: 0.54-1.8; $\mathrm{P}=0.93$ ) (14).

In the largest oncologic study to date, Kronowitz and colleagues reviewed lipofilling in breast reconstruction in 719 cases compared to 670 cases that did not undergo lipofilling and found similar results (16). Recurrence rates were $1.3 \%$ in the AFG group and $2.4 \%$ in the control group $(\mathrm{P}=0.455)$. In a third group comprised of benign breasts treated with prophylactic mastectomy followed by AFG $(n=305)$, the study found no cases of cancer occurrence during the study period. Of note, 33 of these cases were 
prophylactic mastectomies for risk reduction in BRCA mutation carriers. Although the number of patients with BRCA mutations was low in each of these studies, none has demonstrated an increased risk of a cancer eventoccurrence or recurrence-with AFG after mastectomy for BRCA-related risk reduction.

\section{Timing of AFG and recommended technique}

There is no prospective evidence to support a specific timeline for performing AFG in breast reconstruction. Our general practice is to wait to perform AFG until after the patient has completed their oncologic treatment. We do not perform immediate AFG to the breast prior to completion of oncologic treatment, such as at the time of lumpectomy, if radiation therapy is indicated. There is little data to adequately support or refute this practice $(35,36)$; however, the predictability of graft retention remains a challenge in fat grafting. Steady-state graft retention is reported to occur between 2 and 4 months after AFG (28), directly overlapping with the window in which radiation therapy occurs following lumpectomy in BCT. While radiation therapy is effective in addressing residual malignant cells, radiation injury occurs in normal, local-regional tissue as well, making the freshly grafted tissue susceptible to ischemia, fibrosis, and necrosis.

Similarly, with mastectomy and reconstruction, AFG is commonly included with a revision procedure; however, we do not routinely perform AFG at the time of mastectomy so as not to cause more tissue trauma to the mastectomy skin flaps. If radiation therapy is included in the oncologic treatment, we recommend waiting until any acute skin injury has improved before proceeding with AFG. The potential negative effects of radiation such as distortion or fibrosis of the breast may occur up to years following treatment, depending on the modality $(37,38)$. While we feel there may be a benefit to early fat grafting as an adjunct to reconstruction following radiation therapy to mitigate these effects (21), retrospective outcomes are variable and such early grafting ideally would be done in the setting of well-controlled, outcomes-focused studies.

After mastectomy, AFG is used as an adjunct to implantbased or autologous tissue reconstruction as well as AFGonly reconstruction. Regardless of the clinical indication, we have a few standard recommendations to optimize outcomes in fat grafting for breast reconstruction. There are many factors that impact the success of fat grafting, including the quality of the donor and recipient tissues, the amount of contact between the graft and the recipient bed, the presence of ischemia or inflammation, and the transfer of viable cells. If there is scarring or fibrosis of the recipient site, this is first addressed with rigotomy using a small spatulated or forked cannula, with special attention paid to the underlying flap or implant. If the mastectomy skin flaps are thin, recipient site preparation is performed with the delivery cannula as the cannula is inserted.

Delivery of the graft should occur in multiple planes within the subcutaneous and intraglandular spaces. Small aliquots are injected with each pass as the cannula is withdrawn. There should never be a bolus of fat delivered to the recipient site. We prefer a $10-\mathrm{mL}$ syringe with a small-diameter, blunt-tipped cannula. The area of interested should be slightly over-filled to account for partial resorption, but not so much that there will be ischemia and central necrosis. The amount of fat injected is not a set volume but rather determined by how the recipient site feels, which can be particularly challenging in an irradiated field. Fat grafts are not deposited in the same tunnel more than once; instead, a different plane in that location is used. The patient should be counseled pre-operatively that more than one session of AFG may be required to achieve the desired effect, particularly in the setting of irradiated tissue. Our experience, as well as that of others, has shown that complication rates are higher with increasing volume, more sessions of grafting, or a history of radiation therapy $(32,39)$. Soft dressings are used to avoid direct compression of the breasts, and compression garments are worn for donor site support for a minimum of 4 weeks. We encourage gentle massage when moisturizing irradiated skin postoperatively. Finally, a minimum 3- to 6-month interval between grafting sessions is recommended. No imaging is required between sessions.

\section{Recommendations for surveillance and follow-up}

The timing and modality of oncologic follow-up and surveillance should not be affected by AFG in breast reconstruction. A recent survey of a multidisciplinary international panel produced 10 "key" statements regarding AFG based on current evidence and personal experience (40). The panel achieved consensus on the statement "fat grafting is a safe procedure when considering the impact on breast cancer detection and surveillance if a proper technique is used..." However, it is unclear which technique is optimal. We feel that given the radiographic changes that occur with any breast surgery and the subtle findings 
of AFG compared to parenchymal reshaping procedures or BCT, all breast imaging should be reviewed by a breastimaging specialist.

For aesthetic breast surgery (augmentation or asymmetry/contour correction) in which the patient has no personal or family history of breast cancer, standard guidelines for breast cancer screening apply, including age-appropriate mammographic screening and breast self-exams. We recommend pre-operative screening as indicated by age, family history, or abnormal pre-operative exam findings (41). For patients who are 40 years old or older or under the age of 40 with a family history of breast cancer or dense or cystic breasts on physical examination, we recommend screening mammography prior to AFG; however, there does not seem to be a benefit to screening mammography in otherwise healthy women under age 40 with no oncologic family history. In patients over age 40, with dense breasts, or with a family history of breast cancer who undergo screening mammography prior to grafting, annual screening mammography can be subsequently performed as recommended. There is no need to obtain a post-operative imaging study sooner unless there are specific exam findings such as a palpable mass.

In the setting of BCT our current practice is to wait a minimum of 6 months after completion of radiation therapy for follow-up examination by the oncology team and baseline imaging. This is particularly important given the effect that the lumpectomy or oncoplastic reconstruction may have on the mammogram or ultrasound. We have recently sought to answer the question of oncologic safety in partial breast reconstruction via well-defined matched cohorts who underwent BCT with or without AFG at a single cancer center (32). We found no difference in the LRR rate between the two groups with a mean of 6 years of follow-up. There were no significant differences in rates of fat necrosis $(33.3 \%$ versus $34.7 \% ; \mathrm{P}=0.86)$, calcifications $(34.7 \%$ versus $37.5 \% ; \mathrm{P}=0.73)$, or indication for breast biopsy $(22.2 \%$ versus $15.3 \% ; \mathrm{P}=0.23)$ between the two groups and no evidence of interference with cancer surveillance as a result of AFG when imaging was evaluated by a breast specialist. Our study found higher rates of fat necrosis and calcifications in both groups regardless of AFG compared to other studies, pointing to the potential effects of radiation on the remaining breast tissue in the setting of BCT.

Follow-up after AFG for reconstruction following mastectomy should be directed by the oncology team, based on clinical and pathologic characteristics. Most complications from AFG, including a palpable mass, will occur within the early postoperative period. While a mass after grafting is usually benign, it is critical that it be distinguished from malignancy. If there is any suspicion based on examination or imaging, we recommend biopsy. It is our practice to counsel patients pre-operatively on these potential complications of AFG, but to the best of our knowledge, AFG does not translate into an increased risk of recurrence.

\section{Conclusions}

Taken together, the current body of evidence supports the safety and efficacy of AFG in breast reconstruction. These analyses demonstrate a low overall complication rate, high patient and surgeon satisfaction rates, and no evidence that fat grafting promotes cancer recurrence or the development of a new breast cancer. We recommend screening mammography prior to AFG in anyone who is over the age of 40 , has a personal or family history of breast cancer, or has dense breast tissue or other abnormal exam findings. Followup imaging after mastectomy should be guided by the oncology team or based on the reconstructive modality. We recommend a waiting period of 6 months with new baseline imaging for AFG in the setting of BCT. AFG in breast reconstruction does not interfere with oncologic surveillance or imaging; however, there is a need for standardized terminology, protocols, follow-up, and outcome measures in fat grafting as we move forward in the field.

\section{Acknowledgments}

The authors wish to acknowledge the Department of Scientific Publications at the University of Texas MD Anderson Cancer Center for review and assistance with this article.

Funding: None.

\section{Footnote}

Provenance and Peer Review: This article was commissioned by the Guest Editors (Charles E. Butler, Carrie Chu, and Margaret Roubaud) for the series "New Frontiers in Breast Reconstruction" published in Gland Surgery. The article was sent for external peer review organized by the Guest Editors and the editorial office.

Conflicts of Interest: All authors have completed the ICMJE 
uniform disclosure form (available at http://dx.doi. org/10.21037/gs.2020.04.04). The authors have no conflicts of interest to declare.

Ethical Statement: The authors are accountable for all aspects of the work in ensuring that questions related to the accuracy or integrity of any part of the work are appropriately investigated and resolved.

Open Access Statement: This is an Open Access article distributed in accordance with the Creative Commons Attribution-NonCommercial-NoDerivs 4.0 International License (CC BY-NC-ND 4.0), which permits the noncommercial replication and distribution of the article with the strict proviso that no changes or edits are made and the original work is properly cited (including links to both the formal publication through the relevant DOI and the license). See: https://creativecommons.org/licenses/by-ncnd/4.0/.

\section{References}

1. Relations ASoPSP. 2016 Plastic Surgery Stastics Report: ASPS National Clearinghouse of PLastic Surgery Procedural Statistics. 2017.

2. Coleman SR. Structural fat grafting. Aesthet Surg J 1998;18:386-8.

3. Strong AL, Cederna PS, Rubin JP, et al. The Current State of Fat Grafting: A Review of Harvesting, Processing, and Injection Techniques. Plast Reconstr Surg 2015;136:897-912.

4. Hanson SE, Garvey PB, Chang EI, et al. A Prospective Pilot Study Comparing Rate of Processing Techniques in Autologous Fat Grafting. Aesthet Surg J 2019;39:331-7.

5. Eto H, Kato H, Suga H, et al. The fate of adipocytes after nonvascularized fat grafting: evidence of early death and replacement of adipocytes. Plast Reconstr Surg 2012;129:1081-92.

6. Billings E Jr, May JW Jr. Historical review and present status of free fat graft autotransplantation in plastic and reconstructive surgery. Plast Reconstr Surg 1989;83:368-81.

7. Zuk PA, Zhu M, Ashjian P, et al. Human adipose tissue is a source of multipotent stem cells. Mol Biol Cell 2002;13:4279-95.

8. Coppack SW. Pro-inflammatory cytokines and adipose tissue. Proc Nutr Soc 2001;60:349-56.

9. Gutowski KA. Current applications and safety of autologous fat grafts: a report of the ASPS fat graft task force. Plast Reconstr Surg 2009;124:272-80.

10. Rigotti G, Marchi A, Stringhini P, et al. Determining the oncological risk of autologous lipoaspirate grafting for post-mastectomy breast reconstruction. Aesthetic Plast Surg 2010;34:475-80.

11. Petit JY, Lohsiriwat V, Clough KB, et al. The oncologic outcome and immediate surgical complications of lipofilling in breast cancer patients: a multicenter study-Milan-Paris-Lyon experience of 646 lipofilling procedures. Plast Reconstr Surg 2011;128:341-6.

12. Petit JY, Botteri E, Lohsiriwat V, et al. Locoregional recurrence risk after lipofilling in breast cancer patients. Ann Oncol 2012;23:582-8.

13. Cohen O, Lam G, Karp N, et al. Determining the Oncologic Safety of Autologous Fat Grafting as a Reconstructive Modality: An Institutional Review of Breast Cancer Recurrence Rates and Surgical Outcomes. Plast Reconstr Surg 2017;140:382e-92e.

14. Myckatyn TM, Wagner IJ, Mehrara BJ, et al. Cancer Risk after Fat Transfer: A Multicenter Case-Cohort Study. Plast Reconstr Surg 2017;139:11-8.

15. Riggio E, Bordoni D, Nava MB. Oncologic surveillance of breast cancer patients after lipofilling. Aesthetic Plast Surg 2013;37:728-35.

16. Kronowitz SJ, Mandujano CC, Liu J, et al. Lipofilling of the Breast Does Not Increase the Risk of Recurrence of Breast Cancer: A Matched Controlled Study. Plast Reconstr Surg 2016;137:385-93.

17. Kaoutzanis C, Xin M, Ballard TN, et al. Autologous Fat Grafting After Breast Reconstruction in Postmastectomy Patients: Complications, Biopsy Rates, and Locoregional Cancer Recurrence Rates. Ann Plast Surg 2016;76:270-5.

18. Caviggioli F, Maione L, Forcellini D, et al. Autologous fat graft in postmastectomy pain syndrome. Plast Reconstr Surg 2011;128:349-52.

19. Caviggioli F, Maione L, Klinger F, et al. Autologous Fat Grafting Reduces Pain in Irradiated Breast: A Review of Our Experience. Stem Cells Int 2016;2016:2527349.

20. Juhl AA, Karlsson P, Damsgaard TE. Fat grafting for alleviating persistent pain after breast cancer treatment: A randomized controlled trial. J Plast Reconstr Aesthet Surg 2016;69:1192-202.

21. Rigotti G, Marchi A, Galiè M, et al. Clinical treatment of radiotherapy tissue damage by lipoaspirate transplant: a healing process mediated by adipose-derived adult stem cells. Plast Reconstr Surg 2007;119:1409-22.

22. Delay E, Garson S, Tousson G, et al. Fat injection to the 
breast: technique, results, and indications based on 880 procedures over 10 years. Aesthet Surg J 2009;29:360-76.

23. Veber M, Tourasse C, Toussoun G, et al. Radiographic findings after breast augmentation by autologous fat transfer. Plast Reconstr Surg 2011;127:1289-99.

24. Rubin JP, Coon D, Zuley M, et al. Mammographic changes after fat transfer to the breast compared with changes after breast reduction: a blinded study. Plast Reconstr Surg 2012;129:1029-38.

25. Spear SL, Wilson HB, Lockwood MD. Fat injection to correct contour deformities in the reconstructed breast. Plast Reconstr Surg 2005;116:1300-5.

26. Spear SL, Coles CN, Leung BK, et al. The Safety, Effectiveness, and Efficiency of Autologous Fat Grafting in Breast Surgery. Plast Reconstr Surg Glob Open 2016;4:e827.

27. Pinell-White XA, Etra J, Newell M, et al. Radiographic Implications of Fat Grafting to the Reconstructed Breast. Breast J 2015;21:520-5.

28. Illouz YG, Sterodimas A. Autologous Fat Transplantation to the Breast: A Personal Technique with 25 Years of Experience. Aesthetic Plast Surg 2009;33:706-15.

29. Groen JW, Negenborn VL, Twisk JWR, et al. Autologous Fat Grafting in Cosmetic Breast Augmentation: A Systematic Review on Radiological Safety, Complications, Volume Retention, and Patient/Surgeon Satisfaction. Aesthet Surg J 2016;36:993-1007.

30. Groen JW, Negenborn VL, Twisk DJWR, et al. Autologous fat grafting in onco-plastic breast reconstruction: A systematic review on oncological and radiological safety, complications, volume retention and patient/surgeon satisfaction. J Plast Reconstr Aesthet Surg 2016;69:742-64.

31. Flowers CI, Mooney BP, Drukteinis JS. Clinical and imaging surveillance following breast cancer diagnosis. Am Soc Clin Oncol Educ Book 2012:59-64.

32. Hanson SE, Kapur SK, Garvey PB, et al. Oncologic Safety and Surveillance of Autologous Fat Grafting Following

Cite this article as: Hanson SE, Kapur SK, Hwang RF, Dryden MS. Autologous fat grafting in breast reconstruction: implications for follow-up and surveillance. Gland Surg 2021;10(1):487-493. doi: 10.21037/gs.2020.04.04
Breast Conservation Therapy. Plast Reconstr Surg 2020;146:215-25.

33. Agha RA, Fowler AJ, Herlin C, et al. Use of autologous fat grafting for breast reconstruction: A systematic review with meta-analysis of oncological outcomes. J Plast Reconstr Aesthet Surg 2015;68:143-61.

34. Wang K, Dai Y, Pan Y, et al. Local-regional recurrence risk after autologous fat grafting in breast cancer patients: A systematic review and meta-analysis. J Surg Oncol 2020;121:435-40.

35. Biazus JV, Falcao CC, Parizotto AC, et al. Immediate Reconstruction with Autologous fat Transfer Following Breast-Conserving Surgery. Breast J 2015;21:268-75.

36. Stumpf CC, Zucatto ÂE, Cavalheiro JAC, et al. Oncologic safety of immediate autologous fat grafting for reconstruction in breast-conserving surgery. Breast Cancer Res Treat 2020;180:301-9.

37. Youssef A, Stanford J. Hypofractionation Radiotherapy vs. Conventional Fractionation for Breast Cancer: A Comparative Review of Toxicity. Cureus 2018;10:e3516.

38. Correa C, Harris EE, Leonardi MC, et al. Accelerated Partial Breast Irradiation: Executive summary for the update of an ASTRO Evidence-Based Consensus Statement. Pract Radiat Oncol 2017;7:73-9.

39. Herly M, Orholt M, Larsen A, et al. Efficacy of breast reconstruction with fat grafting: A systematic review and meta-analysis. J Plast Reconstr Aesthet Surg 2018;71:1740-50.

40. Nava MB, Blondeel P, Botti G, et al. International Expert Panel Consensus on Fat Grafting of the Breast. Plast Reconstr Surg Glob Open 2019;7:e2426.

41. Lee CH, Dershaw DD, Kopans D, et al. Breast cancer screening with imaging: recommendations from the Society of Breast Imaging and the ACR on the use of mammography, breast MRI, breast ultrasound, and other technologies for the detection of clinically occult breast cancer. J Am Coll Radiol 2010;7:18-27. 